\title{
BALL BEARING VIBRATION MONITORING FOR FAULT DETECTION BY THE ENVELOPE TECHNIQUE
}

\author{
S. B. Alegranzi ${ }^{1}$, J. F. Gonçalves ${ }^{2}$, H. M. Gomes ${ }^{3}$ \\ ${ }^{1}$ Dana Indústrias, Pavilhão A, Distrito industrial, Gravataí, Rio Grande do Sul. \\ ${ }^{2}$ Department of Mechanical Engineering, Federal University of Rio Grande do Sul, \\ ${ }^{3}$ Department of Mechanical Engineering, Federal University of Rio Grande do Sul, \\ (corresponding author: herbert@mecanica.ufrgs.br)
}

\begin{abstract}
This paper presents an application of Hilbert Transform in a method based on vibration analysis by demodulation for identification of bearing failures. This technique is known as Envelope Technique. Initially, a test rig is adjusted and modified in order to acquire the vibration signals from a test bearing under load and rotational speed conditions. Then, experimental tests are performed on ball bearings with induced damage on outer/inner races, which are a very common situation for incipient bearing failure. Several cases are studied, varying the severity/location of the defect and service load condition. Then, it is implemented a software to perform the extraction of the spectrum envelope, that contains the bearing failure signature, using Hilbert transform from the original acceleration waveform. Spectrum envelopes of healthy and defective bearings are compared with the characteristic bearing defect frequencies. The results presented the effectiveness of the method for the studied cases.
\end{abstract}

Keywords: Ball bearing fault detection, Hilbert transform, Envelope spectrum.

\section{INTRODUCTION}

Today's industrial competitiveness demands the companies to seek increasing productivity to stay in the market. This becomes a major problem for the maintenance sector which is responsible to make full use of the mechanical apparatus during their useful service life and also to extend it. Therefore, preventive maintenance techniques become necessary as an alternative to corrective actions that present expressive costs mainly because of unexpected downtimes to repairs. These techniques require systematical inspections based on statistical criteria, manufacturer recommendations, and practical knowledge about the equipment and their operation. It provides information to keep machineries or equipment operating longer at lower overall costs. In order to minimize the downtime for inspections, some conditional techniques can be used, which allow conducting the downtime for repairs only if a defect is detected. This practice is called predictive maintenance and its first step is the defect detection 
which is accomplished by monitoring functional parameters that can indicate certain degradation level. Then, a diagnosis must be established and used to lead to a tendency analysis which can provide statistical information on how long a piece of equipment could operate until collapsing (Mitchell, 1993; Arato Junior, 2004).

The objective of this paper is to present an application of Envelope Technique by using the Hilbert Transform (HT) in a method based on vibration analysis to identify bearing failures.

Faults in rotary components such as bearings, gears, and shafts produce periodical impulse forces which can be easily noticed in the vibration signal. For instance, the identification of the bearing fault occurrence can be done by demodulation of the vibration signal, since the demodulated signal contains the failure signature that depends on which element is affected by the defect. Bearings were used in this work because of its large use and importance in rotary machineries. Although the cost of a bearing is almost negligible when compared with the whole cost of machineries or equipment, a failure occurrence can induce a production interruption causing expressive financial losses (Nepomuceno, 1989).

The demodulation process is performed using a mathematical tool called Hilbert Transform (HT) to extract the envelope of the original acceleration signal. The Hilbert transform is strongly connected with the Fourier transform and its real and imaginary components. Moreover, the HT application can provide information about amplitude, instantaneous phase and frequency of vibration signals. The approach used in this work to evaluate the envelope signal was the phase shift which is made in frequency domain (Randall, 1987).

The experimental data acquisition is performed using a test rig which allows obtaining the acceleration waveform from a test bearing. The service conditions imposed that can be controlled are the shaft rotational frequency and the load applied to the test bearing. Bearings of the same model were tested with no defect and with induced defects on inner/outer races.

It must be emphasized that it was used in this work a very common bearing model. During a maintenance operation, the fault type identification is of minor importance since this bearing model has a low cost. In this situation, for this kind of component, when an imminent fault is detected, the bearing might be simply replaced for a healthy one. Despite this fact, the study proposed here is to characterize the bearing behaviour under an incipient fault condition. This goal is acceptable because the vibration behaviour in the experiments depends on geometrical and functional characteristics, and the formulation is general for all types of ball bearings. For instance, on Eolic generator bearings, which are expensive components, the information about the exact localization of a defect would be extremely necessary to provide suitable conditions to repair.

\section{BIBLIOGRAPHICAL REVIEW}

Vibration analysis is the most used approach to fault detection and its basic premise is that the defect occurrence and propagation result in a modification of the system vibration energy. Time domain techniques are commonly applied to investigate defect progress in rotary components (Arato Junior, 2004). The simplest techniques are based on measurement 
of overall root mean square (RMS) level and crest factor, which is defined as the ratio of peak value to the RMS. This kind of technique can be ineffective to identify the defect sites, though (Bezerra, 2004). Besides that, some statistical parameters such as kurtosis have been used for bearing fault detection. However, both approaches have not been indicated to perform effective detection of incipient fault on bearing elements (Tandon and Choudhury, 1999). The application of a zero-crossing algorithm has been performed, by generating a characteristic feature vector from a measured acceleration waveform, allowing the defect type identification in time domain (William, 2011).

Spectral analysis has been applied to detect the failure signature of defective bearings from the acceleration waveform and has served as a base to several studies. Techniques such as envelope and Cepstrum belong to this frequency domain field. The amplitude-demodulated signal from the acceleration waveform is defined as its envelope and provides innate features about the presence and the exact location of a defect (Bezerra, 2004). The frequency demodulation can also provide reliable information. It has been applied mainly to cases with variations in shaft rotational frequency. An approach using the Teager energy operator to extract the demodulated signals has been proposed in order to suppress the need for filtering steps (Liang, 2010). Cepstrum has been used for bearing fault detection and it allows identifying harmonic peaks family by applying a logarithmic function to the amplitude. This procedure minimizes any influence imposed to the signal during the acquisition. Besides, this highlights the harmonic peaks (Randall, 1987).

Methods that make use of the wavelet transform (WT) are defined as time-frequency domain type and intend to decompose instead of demodulating the vibration signal. Normally, the WT works as a filter and its functional parameters can be optimized by a genetic or a similar optimization algorithm. To highlight the characteristic defect feature, algorithms based on autocorrelation, Fast-Fourier Transform (FFT), Cepstrum etc., are commonly applied, that allow extracting some systematic parameter to be monitored (Nikolaou and Antoniadis, 2002; Su et al, 2010).

\section{BEARING FAILURES}

Bearings are used for rotational displacement guidance, reducing the sliding friction and thus minimizing energy losses, and can be considered the most used mechanical components. Like any component, bearings present degradation of service and the most common causes to the beginning of a defect are: overload, unbalance, sudden temperature changes, inadequate lubrication, incorrect assembly, external vibrations, and manufacturing defects. The incipient fault detection must be directly linked with the observation of potential causes in order to take the necessary steps to mitigate the effective cause. Even if none of these potential causes are presented, bearings are liable for natural fatigue failure. Cyclic shear stresses are generated as the rolling elements move along the race on a loading region, and their consequences can be internal flaws in low resistance points. Over time, these flaws evolve to the race surface where can be transformed into micro-cracks that progress gradually. Normally, a bearing failure spreads slowly and provides signals well ahead of collapsing, which can occur by blocking or rupture of their elements. Typical defects that evolve in this 
way are risks, pitting, and cracks. The process of degradation on bearings can be started on the inner or outer race, one of the rolling elements, or cage and can spread to the remaining elements generating very specific and predictable characteristics (Nepomuceno, 1989; Juvinall and Marshek, 1991).

When a defective surface of a bearing element gets in contact with the rolling elements, an impulse is generated, periodically producing a harmonic series with a fundamental frequency that depends on which surface the defect occurred. Considering that the inner race turns at the shaft rotational frequency and the outer race is fixed, an inner race defect would be localized in a moving point with relation to the loading region. Therefore, the impulse series generated will be modulated by a signal proportional to the loading. On the other hand, for an outer race defect, the impulse series will be generated only if the defective point is located on a loading region. In any case, the defect characteristic frequencies are given by the following equations:

$$
f_{o}=f_{r} \frac{N}{2}\left(1-\frac{d_{b} \cos \phi}{d_{p}}\right), \quad f_{i}=f_{r} \frac{N}{2}\left(1+\frac{d_{b} \cos \phi}{d_{p}}\right), \quad f_{b}=f_{r} \frac{d_{p}}{d_{b}}\left(1-\frac{d_{b}{ }^{2} \cos ^{2} \phi}{d_{p}{ }^{2}}\right)
$$

where $f_{o}$ is the outer race defect characteristic frequency, $f_{i}$ is the inner race defect characteristic frequency, $f_{b}$ is the ball defect characteristic frequency, $f_{r}$ is the shaft rotational frequency, $N$ is the number of rolling elements, $d_{b}$ is the ball diameter, $d_{p}$ is the pitch diameter, and $\phi$ is the ball contact angle. If a defect occurs on the bearing cage, the cage defect characteristic frequency $\left(f_{c}\right)$ will be conspicuous. This frequency is given by the following expression:

$$
f_{c}=f_{r} \frac{1}{2}\left(1+\frac{d_{b} \cos \phi}{d_{p}}\right)
$$

The cage defect characteristic frequency is important to characterize not only a defect on the cage but also a defect on a rolling element. It occurs because a defect on a ball, which has its movement restricted by the cage, would incite impulses on that frequency. The geometrical parameters used to obtain the defect characteristic frequencies are shown in Fig. 1. Equations (1) and (2) were obtained for bearings with ball rolling elements and considering that there is rolling contact, but not sliding, between the elements (Mitchell, 1993).
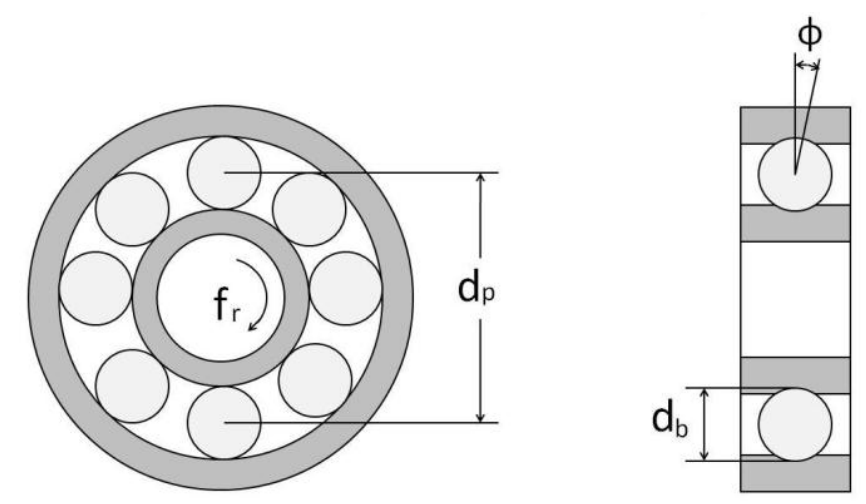

Figure 1. Ball bearing dimension definitions. 


\subsection{Hilbert transform}

The Hilbert transform (HT) describes the connection between the real and imaginary components of the Fourier Transform of a one-sided signal, for instance the impulse response of a causal system. For a real signal $x(t)$, its Hilbert transform can be defined as the convolution integral, between $x(t)$ and $(1 / \pi t)$, expressed by the following equation:

$$
\mathcal{H}\{x(t)\}=\frac{1}{\pi} \int_{-\infty}^{\infty} \frac{x(\tau)}{t-\tau} d \tau
$$

The inverse HT can also be defined as a convolution integral, between $x(t)$ and $(-1 / \pi t)$. It is explicit that a singularity can occur when $t=\tau$, so the integral must be considered as a Cauchy principal value.

In that case, the HT of a real signal in time domain $x(t)$ is another real signal in time domain, defined as $\mathcal{H}\{x(t)\}=\tilde{x}(t)$, so that the signal given by $z(t)=x(t)+i \tilde{x}(t)$ is an analytic signal which can be written as:

$$
z(t)=A(t) e^{i \theta(t)}
$$

where $A(t)$ is the envelope of $x(t)$, and $\theta(t)$ is the phase function. In frequency domain, the HT can be defined as a phase shift, by the following form:

$$
\theta(f)=\mathfrak{F}\{\theta(t)\} \theta(f) \rightarrow \theta(f)+\frac{\pi}{2} \text { for } f>0 \text { and } \theta(f) \rightarrow \theta(f)-\frac{\pi}{2} \text { for } f<0
$$

The most common application of HT is to perform the demodulation of amplitude modulated (AM) signals in digital way. An AM signal is formed by the arrangement of a carrier signal and a modulator signal, as shown in Fig. 2. In this example, the modulation index, which relates the amplitudes of modulator and carrier signals, is 0.667 . Moreover, the fundamental frequencies of the carrier and modulator signals also compose the spectrum of AM signal (Haykin, 1989; Randall, 1987).

The objective of demodulation is to extract the envelope from a modulated signal, as shown in Fig. 2. However, it is important not to disarray the modulator signal of an AM signal with the envelope of an AM signal, which can be simply explained as a contour curve formed by connecting the peaks of amplitude.

It must be clarified that the ideal HT as a phase shifter may not be performed perfectly to experimental signals, especially under effect of noises. This digital tool has its performance harmed and the experimental signal must be physically filtered to minimize these process issues (Proakis, 1988; Feldman, 2011).

\section{EXPERIMENTAL METHODOLOGY}

The test rig was fixed over a vibration desk which isolates the rig base from external vibration interferences, and is shown in Fig. 3. The rotation is transmitted to the shaft by a pulley-belt system with a 1HP induction motor, and can be controlled by a frequency driver inverter to impose the required shaft rotational frequency. The test bearing is the component where the ball bearing is assembled to be tested. This bearing is composed by two connection pins to attach the radial and axial accelerometers, and one connection pin to attach the load cell. 


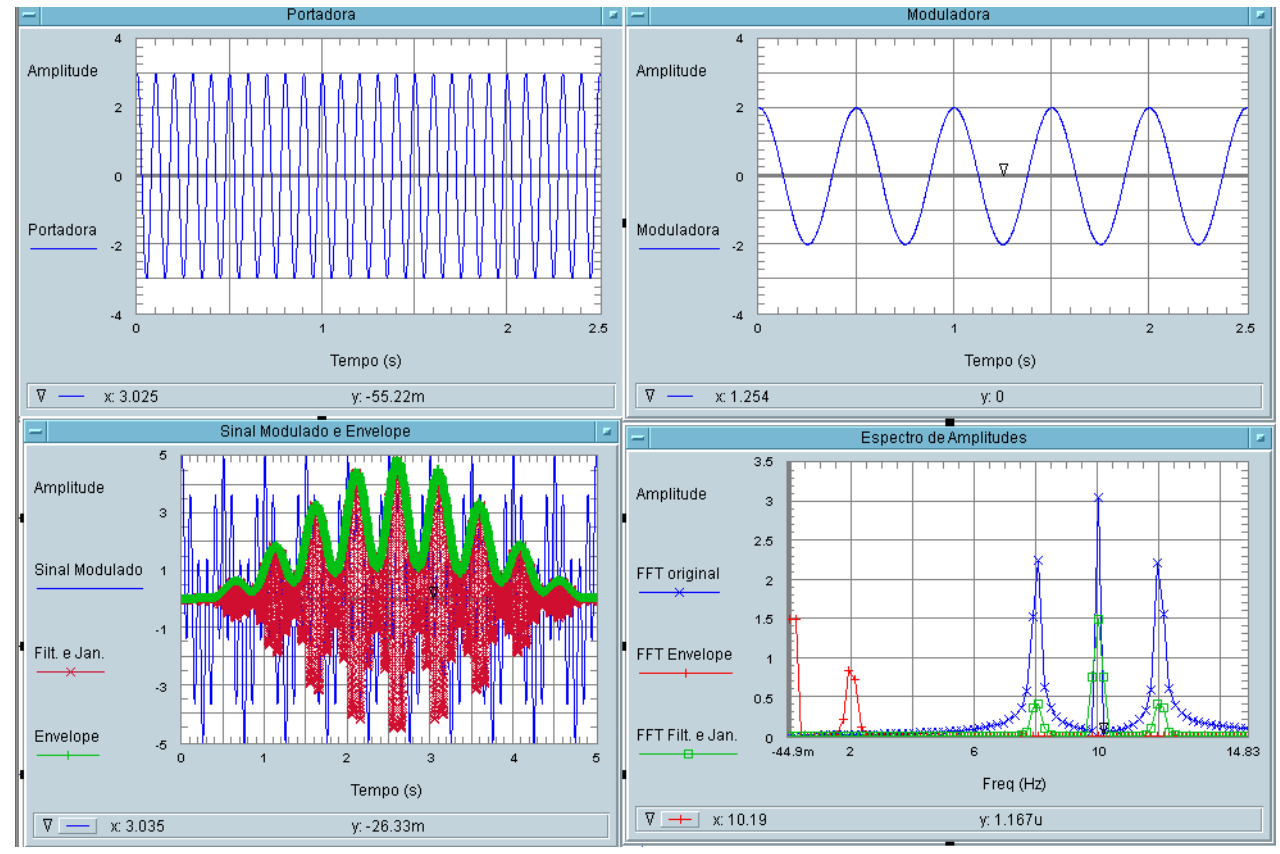

Figure 2. Demodulation of a vibration signal; (a) carrier signal $3 \cos (2 \pi 10)$; (b) modulator signal $2 \cos (2 \pi 2)$; (c) modulated signal, windowed/filtered signal and envelope of the windowed signal; (d)Original signal, envelope signal and windowed/filtered signal Fourier transforms.



1 - Damped base; 2 - electric induction motor; 3 -Pulley-belt transmission system; 4 - Shaft; 5 -Journal bearings; 6 -Tested journal bearing; 7 - Accelerometer for data acquisition; 8 - Load cell; 9 -Tested ball bearing;

Figure 3. Ball bearing test rig.

The envelope technique software was implemented in order to analyze the modifications on the vibration level in system resonant frequencies, and the basic steps are shown in Fig. 4. Initially, the original signal that is acquired by accelerometers is windowed using the Hanning function to minimize the leakage effects. This phenomenon occurs during the fast-Fourier transformation of a signal with no integer number of cycles, and can induce 
distortions in spectrum analysis because of the energy dispersing effect. Then, the FFT is applied to lead the signal to the frequency domain and a pass-band filter is imposed to extract the system resonant frequencies. The IFFT transforms the signal back to time domain where the envelope is evaluated by a phase shift conditional command according to the Eq. (5). Finally, the FFT is applied to the envelope signal to highlight the defect frequencies $\left(f_{d}\right)$ on spectrum analysis.

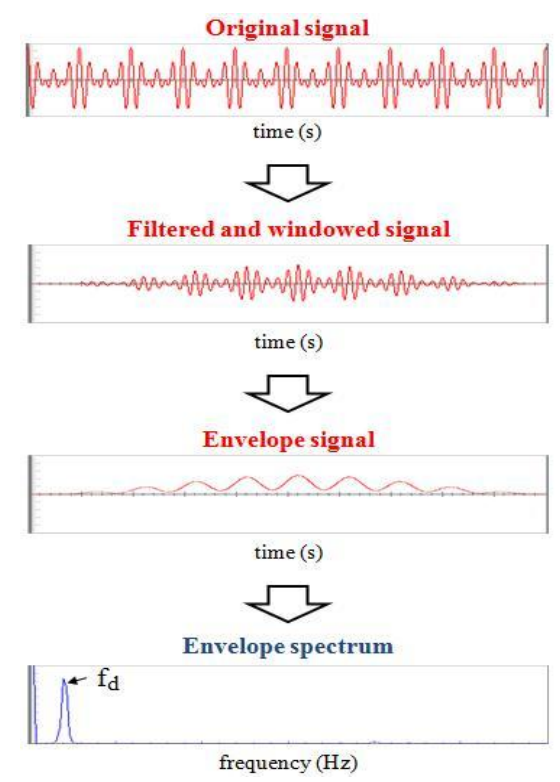

Figure 4. Basic steps for Envelope Technique procedure.

The defect frequency $\left(f_{d}\right)$ found in envelope spectrum analysis must be compared with the defect characteristic frequencies to identify the type of defect. However, other characteristics such as side-bands and harmonics must be analyzed in order to identify correctly the defect type. These particular features appear depending on which bearing element the defect is induced.

The relevant data regarding the tested ball bearings are shown in Table 1. All these parameters were obtained in the manufacturer's handbook.

Table 1. Test bearing data.

\begin{tabular}{c|c|c|c|c}
\hline Model & $d_{p}(\mathrm{~mm})$ & $d_{b}(\mathrm{~mm})$ & $N$ & $\begin{array}{c}\phi \\
(\mathrm{rad})\end{array}$ \\
\hline \hline 6204 & 33.50 & 7.938 & 8 & 0.14 \\
\hline
\end{tabular}

The defects were induced using EDM (electroerosion). For this experimental study, the defects were induced on eight ball bearings while one was kept healthy. Four of them were induced in the outer race and the other four in the inner race. The defect dimensions were selected for equal deep but increasing width, so the ball will fall in the fault with increasing heights. The defects were for the outer race, the ball falling height $\mathrm{x}$ fault width were $0.33 \mathrm{~mm}$ x $3.5 \mathrm{~mm} ; 0.24 \mathrm{~mm} \times 3.0 \mathrm{~mm} ; 0.1 \mathrm{~mm} \times 2.0 \mathrm{~mm} ; 0.03 \mathrm{~mm} \times 1.0 \mathrm{~mm}$. The defects were for the inner race $0.53 \mathrm{~mm} \times 3.5 \mathrm{~mm} ; 0.38 \mathrm{~mm} \times 3.0 \mathrm{~mm} ; 0.17 \mathrm{~mm} \times 2.0 \mathrm{~mm} ; 0.04 \mathrm{~mm} \times 1.0 \mathrm{~mm}$. Figure 5 (a) and (b) present the defective inner race and outer race.

The experiments were performed under loading conditions of $2500 \mathrm{~N}$ and $3750 \mathrm{~N}$. It is expected that the load applied to the bearing has influence on the amplitude of the peaks of acceleration in time domain and thus in the spectrum but there is no relation between the loading condition and the defect characteristic frequencies. Therefore, the tests were 
established as described in Table 2 only for the $1795 \mathrm{rpm}$ condition and at $2500 \mathrm{~N}$ and $3750 \mathrm{~N}$. Three samples were recorded for each of the ball bearing defects. The tests were conducted first for the outer race defects and afterwards for the inner race defect. The falling heights in both cases were in decreasing order. The acceleration waveforms of the healthy and defective bearings were collected through data acquisition software at a sampling rate of $22.5 \mathrm{kHz}$.

(a)

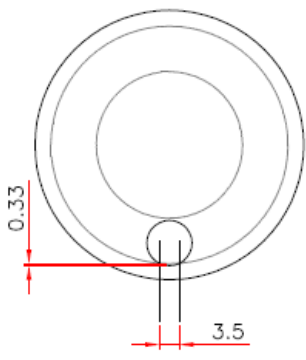

(b)

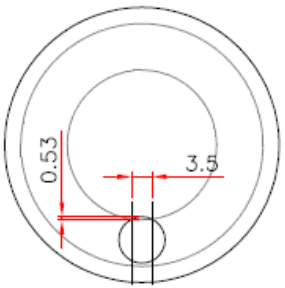

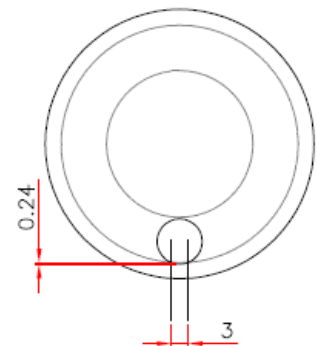

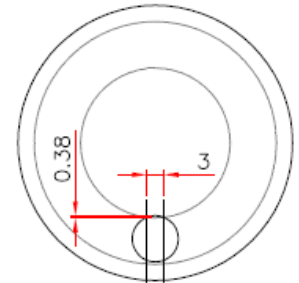

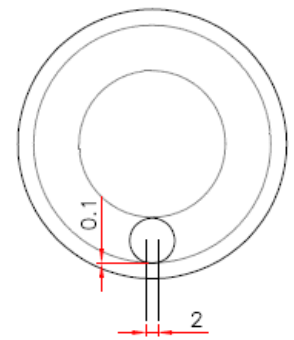

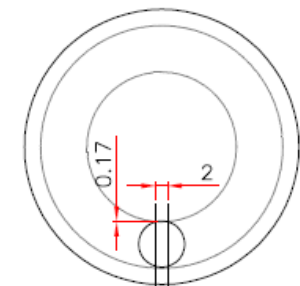

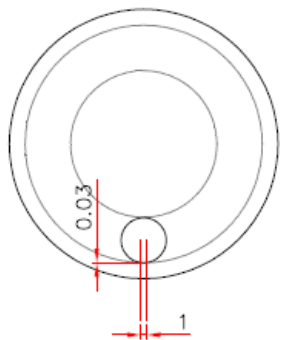

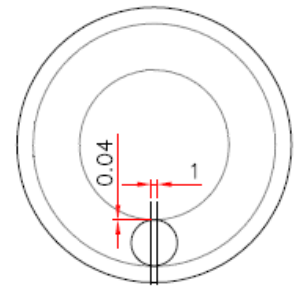

Figure 5. Induced defects: (a) decreasing fault width in outer race with increasing ball falling heights, (b) decreasing fault width in inner race with increasing ball falling heights).

\section{RESULTS}

The spectrums obtained by the Envelope Technique, according to the procedure shown in Fig. 4, are presented and analyzed in this section. The defect frequency $\left(f_{d}\right)$ highlighted in the spectrum is compared with the defect characteristic frequencies shown in Table 3 . It was evaluated according to Eq. (1) and Eq. (2). The results presented in Table 3 reflect the mean values of the several samples.

Typical envelope spectrum from the healthy bearing (bearing R0) is presented in Fig. 6. No pronounced peaks were found in the spectrum of the envelope signal. For R1 to R4 tests, which were carried out using a bearing with damage induced on the outer race, the spectrum of the envelope allows noticing peaks equally spaced. A typical spectrum is presented in Fig. 7. The first peak is the outer race defect characteristic frequency $\left(f_{o}\right)$ which is followed by its harmonics $\left(2 f_{o} ; 3 f_{o} ; 4 f_{o}\right)$. These failure signatures are typically related to cases where the defect occurs in a point of the race which has no movement regarding the loading region. It is caused because the periodical impulse series excite the 
system resonant modal on the characteristic frequency of this kind of defect.

Table 2. Experimentally tested conditions.

\begin{tabular}{l|l|l|l}
\hline Test & Defect location & $n(\mathrm{rpm})$ & Defect \\
\hline \hline R0 & No defect & 1795 & No defect \\
\hline R1 & Outer race & 1795 & $0.33 \mathrm{~mm} \times 3.5 \mathrm{~mm}$ \\
\hline R2 & Outer race & 1795 & $0.24 \mathrm{~mm} \times 3.0 \mathrm{~mm}$ \\
\hline R3 & Outer race & 1795 & $0.10 \mathrm{~mm} \times 2.0 \mathrm{~mm}$ \\
\hline R4 & Outer race & 1795 & $0.03 \mathrm{~mm} \times 1.0 \mathrm{~mm}$ \\
\hline R5 & Inner race & 1795 & $0.53 \mathrm{~mm} \times 3.5 \mathrm{~mm}$ \\
\hline R6 & Inner race & 1795 & $0.38 \mathrm{~mm} \times 3.0 \mathrm{~mm}$ \\
\hline R7 & Inner race & 1795 & $0.17 \mathrm{~mm} \times 2.0 \mathrm{~mm}$ \\
\hline R8 & Inner race & 1795 & $0.04 \mathrm{~mm} \times 1.0 \mathrm{~mm}$ \\
\hline
\end{tabular}

Table 3. Mean defect characteristic frequencies obtained experimentally.

\begin{tabular}{c|c|c}
\hline$f_{r}(\mathrm{~Hz})$ & $f_{o}(\mathrm{~Hz})$ & $f_{i}(\mathrm{~Hz})$ \\
\hline \hline 30 & 91.3 & 148.00 \\
\hline R0 & - & - \\
\hline R1 & 96.11 & - \\
\hline R2 & 96.67 & - \\
\hline R3 & 96.78 & - \\
\hline R4 & 96.78 & - \\
\hline R5 & - & 154.67 \\
\hline R6 & - & 154.00 \\
\hline R7 & - & 154.00 \\
\hline R8 & - & 153,23 \\
\hline
\end{tabular}

In contrast, the spectra of the envelopes of tests R5 to R8, which a typical graph is shown in Fig. 8, present the failure signature for inner race defect. The defect characteristic frequency $\left(f_{i}\right)$ is followed and anteceded by side-bands spaced of the shaft rotational frequency $\left(f_{r}\right)$, that is the modulator signal frequency, as previously defined in Fig. 2 . It occurs because the periodical impulse series (at frequency $f_{i}$ ) is modulated by the load magnitude (at frequency $f_{r}$ ), which vary as the shaft turns.

Table 4 shows the results for fault in the outer race and vertical accelerometer. Table 5 shows the results for fault in the inner race and vertical accelerometer.

It was found little correlation relating the magnitude of the spectrum of the envelope with the severity of the damage. This maybe is due to the fact that the wider is the induced defect, the looser the ball stays on the defect making the surrounding balls to carry and extra load from the central ball. More experimental investigations should be conducted in order to clarify this behavior. 


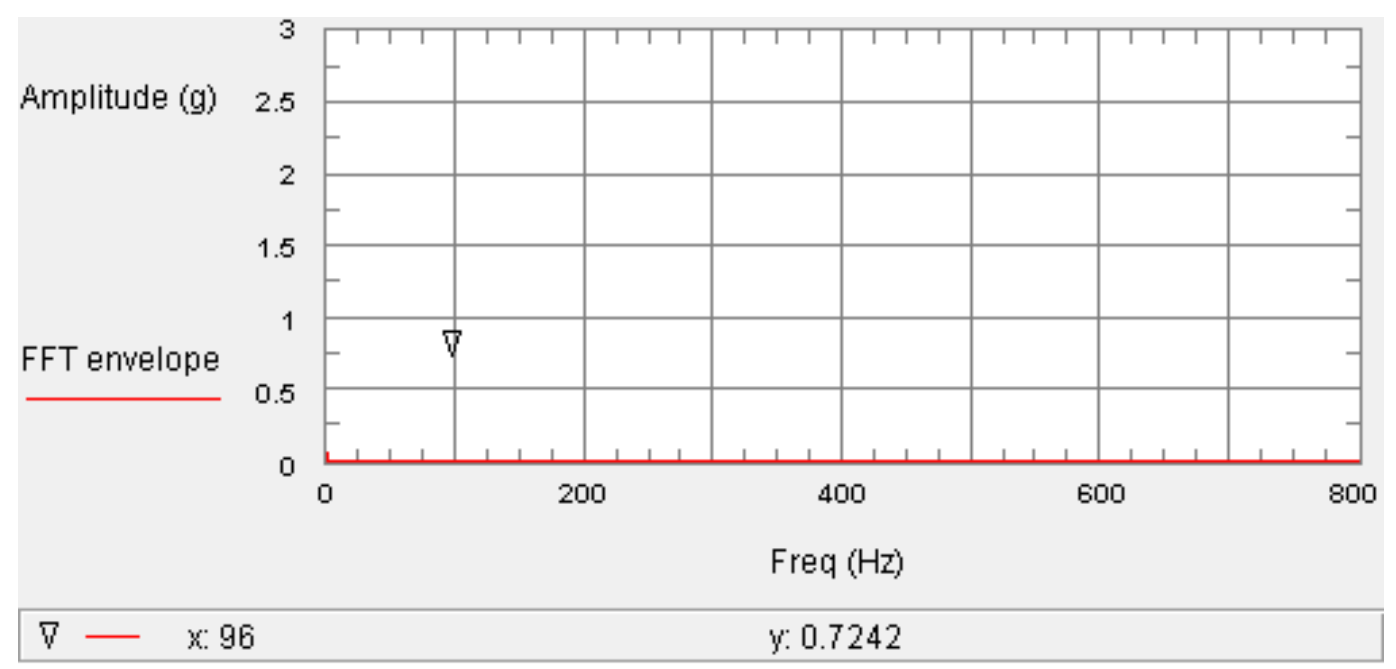

Figure 6. Typical envelope spectrum of the vibration signal for test R0 (healthy ball bearing).

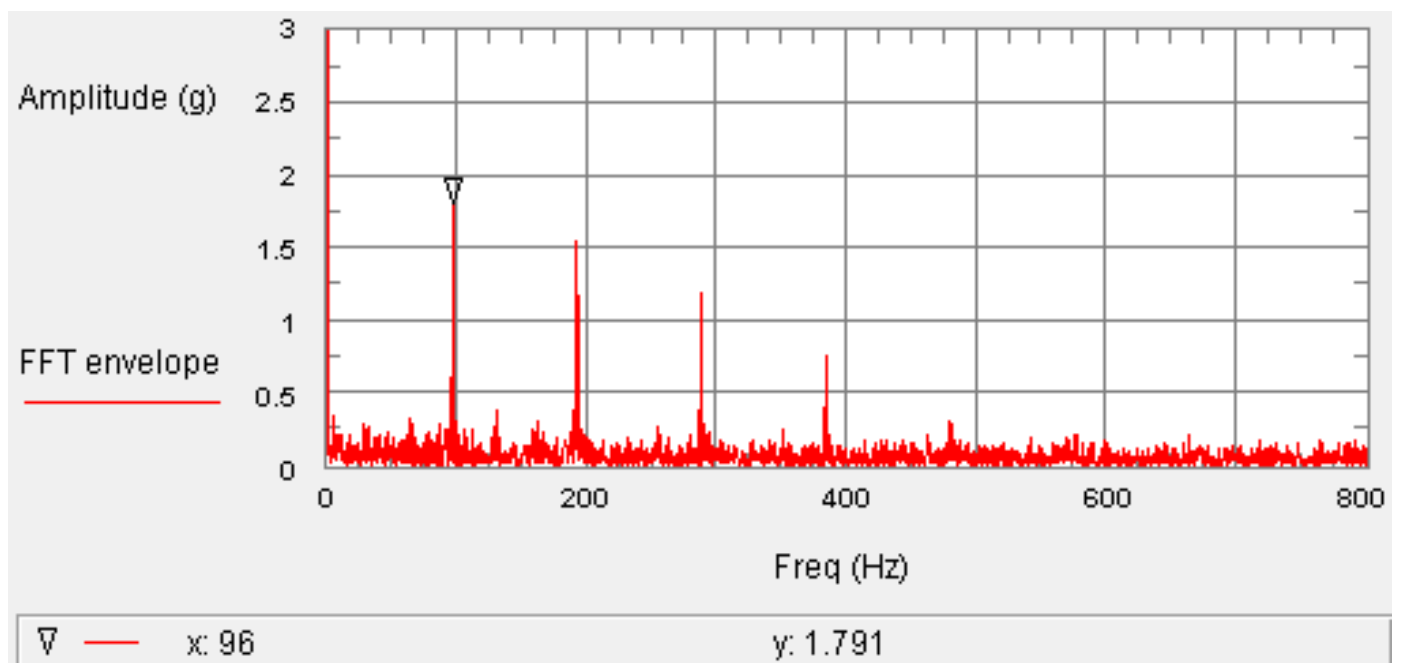

Figure 7. Typical envelope spectrum of the vibration signal for tests R1-R4 (outer race fault).

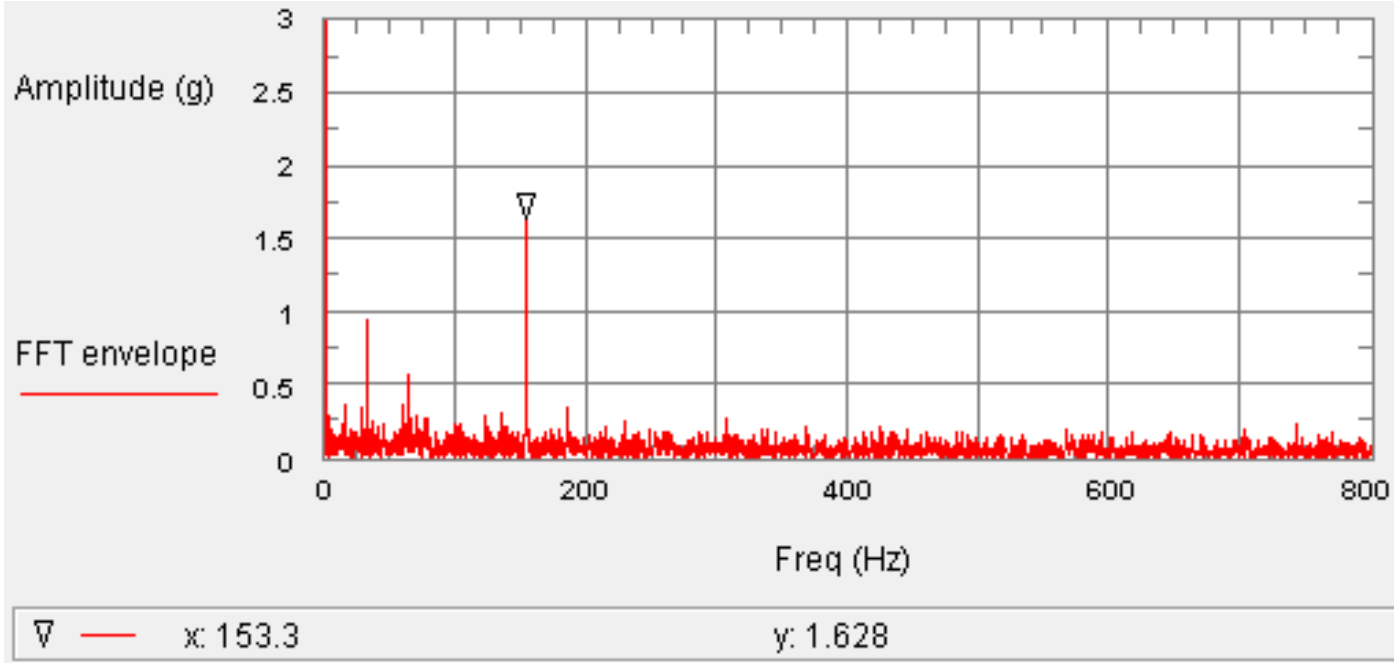

Figure 8. Typical envelope spectrum of the vibration signal for tests R5-R8 (inner race fault). 
Table 4. Amplitude spectrum for outer race fault and vertical accelerometer.

\begin{tabular}{|c|c|c|c|c|c|c|}
\hline Bearing & $\begin{array}{c}\text { Service } \\
\text { Load }\end{array}$ & Sample & Amplitude & $\begin{array}{c}\text { Experimentally } \\
\text { obtained fault } \\
\text { frequency } \\
{[\mathrm{Hz}]} \\
\end{array}$ & $\begin{array}{c}\text { Analytical Fault } \\
\text { Frequency } \\
{[\mathrm{Hz}]}\end{array}$ & $\begin{array}{c}\text { Difference } \\
{[\%]}\end{array}$ \\
\hline \multirow{6}{*}{ R1 } & \multirow{3}{*}{1} & 1 & 2,000 & 96,00 & 91,3 & $5,14 \%$ \\
\hline & & 2 & 4,222 & 96,33 & 91,3 & $5,50 \%$ \\
\hline & & 3 & 3,886 & 96,00 & 91,3 & $5,14 \%$ \\
\hline & \multirow{3}{*}{2} & 1 & 4,572 & 96,00 & 91,3 & $5,14 \%$ \\
\hline & & 2 & 0,559 & 95,67 & 91,3 & $4,77 \%$ \\
\hline & & 3 & 0,867 & 96,33 & 91,3 & $5,50 \%$ \\
\hline \multirow{6}{*}{$\mathrm{R} 2$} & \multirow{3}{*}{1} & 1 & 2,398 & 96,33 & 91,3 & $5,50 \%$ \\
\hline & & 2 & 0,542 & 95,33 & 91,3 & $4,40 \%$ \\
\hline & & 3 & 4,955 & 96,33 & 91,3 & $5,50 \%$ \\
\hline & \multirow{3}{*}{2} & 1 & 5,385 & 96,67 & 91,3 & $5,87 \%$ \\
\hline & & 2 & 4,741 & 96,67 & 91,3 & $5,87 \%$ \\
\hline & & 3 & 2,820 & 96,67 & 91,3 & $5,87 \%$ \\
\hline \multirow{6}{*}{$\mathrm{R} 3$} & \multirow{3}{*}{1} & 1 & 2,760 & 96,67 & 91,3 & $5,87 \%$ \\
\hline & & 2 & 1,258 & 96,67 & 91,3 & $5,87 \%$ \\
\hline & & 3 & 0,341 & 98,67 & 91,3 & $8,06 \%$ \\
\hline & \multirow{3}{*}{2} & 1 & 2,627 & 96,67 & 91,3 & $5,87 \%$ \\
\hline & & 2 & 1,167 & 96,67 & 91,3 & $5,87 \%$ \\
\hline & & 3 & 1,705 & 96,67 & 91,3 & $5,87 \%$ \\
\hline \multirow{6}{*}{$\mathrm{R} 4$} & \multirow{3}{*}{1} & 1 & 0,340 & 96,33 & 91,3 & $5,50 \%$ \\
\hline & & 2 & 2,973 & 96,33 & 91,3 & $5,50 \%$ \\
\hline & & 3 & 3,129 & 96,33 & 91,3 & $5,50 \%$ \\
\hline & \multirow{3}{*}{2} & 1 & 0,761 & 96,00 & 91,3 & $5,14 \%$ \\
\hline & & 2 & 2,832 & 96,00 & 91,3 & $5,14 \%$ \\
\hline & & 3 & 2,298 & 96,33 & 91,3 & $5,50 \%$ \\
\hline
\end{tabular}

Table 5. Amplitude spectrum for inner race fault and vertical accelerometer.

\begin{tabular}{|c|c|c|c|c|c|c|}
\hline Bearing & $\begin{array}{l}\text { Service } \\
\text { Load }\end{array}$ & Sample & Amplitude & $\begin{array}{c}\text { Experimentally } \\
\text { obtained fault } \\
\text { frequency } \\
{[\mathrm{Hz}]} \\
\end{array}$ & $\begin{array}{l}\text { Analytical Fault } \\
\text { Frequency } \\
\text { [Hz] }\end{array}$ & $\begin{array}{c}\text { Difference } \\
{[\%]}\end{array}$ \\
\hline \multirow{6}{*}{ R5 } & \multirow{3}{*}{1} & 1 & 1,745 & 154,00 & 148,0 & $4,04 \%$ \\
\hline & & 2 & 1,485 & 156,00 & 148,0 & $5,39 \%$ \\
\hline & & 3 & 1,524 & 154,30 & 148,0 & $4,24 \%$ \\
\hline & \multirow{3}{*}{2} & 1 & 2,869 & 153,30 & 148,0 & $3,57 \%$ \\
\hline & & 2 & 2,559 & 153,30 & 148,0 & $3,57 \%$ \\
\hline & & 3 & 2,637 & 153,30 & 148,0 & $3,57 \%$ \\
\hline \multirow{6}{*}{ R6 } & \multirow{3}{*}{1} & 1 & 1,446 & 154,00 & 148,0 & $4,04 \%$ \\
\hline & & 2 & 3,222 & 154,00 & 148,0 & $4,04 \%$ \\
\hline & & 3 & 1,490 & 154,00 & 148,0 & $4,04 \%$ \\
\hline & \multirow{3}{*}{2} & 1 & 1,419 & 154,30 & 148,0 & $4,24 \%$ \\
\hline & & 2 & - & - & 148,0 & - \\
\hline & & 3 & 1,418 & 153,70 & 148,0 & $3,84 \%$ \\
\hline \multirow{6}{*}{ R7 } & \multirow{3}{*}{1} & 1 & 1,503 & 154,30 & 148,0 & $4,24 \%$ \\
\hline & & 2 & 2,415 & 153,70 & 148,0 & $3,84 \%$ \\
\hline & & 3 & 2,722 & 153,70 & 148,0 & $3,84 \%$ \\
\hline & \multirow{3}{*}{2} & 1 & 0,797 & 153,70 & 148,0 & $3,84 \%$ \\
\hline & & 2 & 0,487 & 154,00 & 148,0 & $4,04 \%$ \\
\hline & & 3 & 2,511 & 153,70 & 148,0 & $3,84 \%$ \\
\hline \multirow{6}{*}{$\mathrm{R} 8$} & \multirow{3}{*}{1} & 1 & 0,436 & 154,00 & 148,0 & $4,04 \%$ \\
\hline & & 2 & 1,295 & 154,00 & 148,0 & $4,04 \%$ \\
\hline & & 3 & 0,857 & 154,00 & 148,0 & $4,04 \%$ \\
\hline & \multirow{3}{*}{2} & 1 & 1,047 & 154,00 & 148,0 & $4,04 \%$ \\
\hline & & 2 & 1,590 & 154,00 & 148,0 & $4,04 \%$ \\
\hline & & 3 & 1,697 & 154,00 & 148,0 & $4,04 \%$ \\
\hline
\end{tabular}




\section{FINAL REMARKS}

Some initial results were presented in this work dealing with the problem of defect type identification on ball bearings. A review of the issues addressed in this paper such as the bearing failure characterization and the Hilbert Transform (HT) definitions was briefly presented, and a methodology to acquire the experimental data derived from the bearing vibration and to extract the envelope of the acceleration waveform was proposed.

For the demodulation, an algorithm using the HT as a phase shifter in frequency domain was used. This process is very suitable to obtain the signal envelopes of discrete acceleration waveforms.

The failure signatures were easily noticed in the envelope spectra and also the modulation process that occurs when certain types of defect are present in this kind of rotary component. Moreover, the average differences between the defect characteristic frequencies analytically obtained and the defect frequencies highlighted in the envelope spectra was about $5.0 \%$ showing good correlation with experimental investigation. However, small discrepancies occur because some assumptions, such as the low ball contact angle and no sliding contact between elements were considered to evaluate these frequencies.

These preliminary results show that the implementation was successfully accomplished: the HT algorithm was implemented, the test rig apparatus presented adequate for the experiments and the collected experimental data validate the theory. It is concluded that the method is promising in incipient damage detection on bearings.

It was not possible to find a correlation or trend between the magnitudes of the spectrum of the envelope and the fault severity. This is supposed to be due to the fact that the wider is the induced defect, the looser the ball stays on the defect making the surrounding balls to carry the remaining load for the central ball, reducing the impact. More experimental investigations should be conducted in order to clarify this behavior.

Future works would involve different defect types, such as cage defect, ball defects, multiple defects and a larger number of tests could be executed in order to perform a statistical analysis. Improvements in the data processing system to provide the failure evolution monitoring, as well as a real-time graphical interface, could also be improved.

\section{Acknowledgements}

The authors are grateful for the support received from the CNPq and CAPES.

\section{REFERENCES}

[1] Arato Junior, A. "Manutenção preditiva usando análise de vibrações". Barueri: Manole, 2004.

[2] Bezerra, R. A. "Detecção de falhas em rolamentos por análise de vibração". D.Sc. Thesis, FEM, Universidade Estadual de Campinas, Campinas, Brasil, 2004.

[3] Feldman, M. "Hilbert Transform in vibration analysis". Mechanical systems and signal processing, 25:735-802, 2011.

[4] Haykin, S. "An introduction to analog and digital communication". New York: John Wiley \& Sons, 1989.

[5] Juvinall, R. C. and Marshek, K. M. "Fundamentals of machine component design", $2^{\text {nd }}$ edition. New York: John Wiley \& Sons, 1991. 
[6] Liang, M. and Bozchalooi, I. S. "An energy operator approach to joint application of amplitude and frequency-demodulations for bearing fault detection". Mechanical systems and signal processing, 24:1473-1494, 2010.

[7] Mitchell, J. S. "Introduction to machinery analysis and monitoring", $2^{\text {nd }}$ edition. Oklahoma: Penn Well Books, 1993.

[8] Nepomuceno, L. X. “Técnicas de manutenção preditiva”. São Paulo: Editora Edgard Blücher, 1989.

[9] Nikolaou, N. G. and Antoniadis, I. A. "Rolling element bearing fault diagnosis using wavelet packets". NDT\&E International, 35: 197-205, 2002.

[10] Proakis, J. G. and Manolakis, D. G. "Introduction to digital signal processing". New York: Macmillan, 1988.

[11] Randall, R. B. "Frequency analysis", $3^{\text {rd }}$ edition. Naerum: Brüel \& Kjaer, 1987.

[12] Su, W., Wang, F., Zhu, H., Zhang, Z. and Guo, Z. "Rolling element bearing faults diagnosis based on optimal Morlet wavelet filter and autocorrelation enhancement". Mechanical systems and signal processing, 24:1458-1472, 2010.

[13] William, P. E. and Hoffman, M. W. "Identification of bearing faults using time domain zero-crossings". Mechanical systems and signal processing, 25:1096-1216, 2011. 